\title{
Second versus first wave of COVID-19 in patients with MPN
}

(c) The Author(s), under exclusive licence to Springer Nature Limited 2022

Leukemia (2022) 36:897-900; https://doi.org/10.1038/s41375-02201507-2

\section{TO THE EDITOR}

The first wave of the SARS-CoV-2 coronavirus disease 2019 (COVID19) began in January 2020, affecting many European countries and leading to an overwhelming of the capacity of acute care hospitals and intensive care units (ICUs). Patients with hematologic malignancies incurring COVID-19 were among the most vulnerable [1-3] and in those with myeloproliferative neoplasms (MPN) including essential thrombocythemia (ET), polycythemia vera (PV), prefibrotic myelofibrosis (pre-PMF) and myelofibrosis (MF), deaths were registered in $28 \%$ of cases, being particularly elevated in MF (48\%). Age, male gender, admission to ICU, severity of COVID-19 and ruxolitinib discontinuation at COVID-19 diagnosis were independent risk factors for death [4].

The pandemic substantially subsided in Europe until October 2020, likely due to non-pharmaceutical control measures including wearing of a mask, hand washing, social distancing, quarantine and city/region lockdown. These interventions gradually relaxed in consideration of the trade-off between economic sustainability and public health, leading to a second wave of infection, also triggered by new SARS-CoV-2 variants. These raised concerns and uncertainties regarding a possibly new clinical epidemiology of the new virus variants in terms of presentation, severity of acute infection and clinical outcomes.

In the present analysis, we report the outcomes recorded in the 12 months after the first wave declined, pursuing a dual purpose: (i) to describe possible differences of COVID-19 presentation between the two waves and (ii) to evaluate the rate and risk factors of relevant outcomes, including mortality, thrombosis and main clinical events in MPN patients surviving after the acute phase of COVID-19.

The MPN-COVID study is steadily enrolling consecutive adult MPN patients with COVID-19 infection since February 15, 2020. Thirty-nine hematologic centers from Italy, Spain, Germany, France, UK, Poland, and Croatia enrolled 175 and 304 cases in the first and second wave, respectively.

In the present analysis, we report data of the second wave of the pandemic, from July 1, 2020 to June 30, 2021, and compare findings with those obtained during the first wave (i.e., from February 15 to June 30, 2020).

\section{INCIDENCE OF MPN-COVID CASES AND PROBABILITY DENSITY OF DEATH}

Supplementary Fig. 1S illustrates the distribution probability of incidence and density of COVID-19 cases by Kernel method [5] for to the two pandemic periods. During the first wave, a peak was documented from April to May followed by a decline during the summer season, whereas the second wave peaked in November/ December 2020 and did not completely decline until June 2021. The shape of the incidence curve was substantially similar, while the density function of deaths was less pronounced in the second wave.

\section{PRESENTATION AND THERAPY}

In comparison with the first, patients in the second wave were younger, had with less comorbidities and presented with moderate COVID-19 infection (Table 1). They were less symptomatic, most were treated at home, intensive respiratory support being required in a limited number of cases, and an elevation of blood inflammatory markers (C-Reactive Protein and Neutrophil to Lymphocyte Ratio) was found in a lower proportion of cases.

In regard to COVID-19 and MPN directed therapy, steroids were more frequently prescribed than in the first wave $(p=0.007)$; conversely, ruxolitinib was discontinued in fewer MF hospitalized patients. Therefore, all of these clinical and laboratory data were consistent with a less severe COVID-19 infection.

\section{MORTALITY AND RELATED RISK FACTORS}

Survival during the first vs. second wave (69\% vs. $91 \%)$ at 60 days after COVID-19 diagnosis, was statistically different $(p<0.001)$ (Fig. 1A). Among 26 deaths registered during the second wave, 4 (15\%) occurred at home, $19(73 \%)$ on the regular word and $3(12 \%)$ in the ICU, and occurred in MF $(n=17,65 \%), \mathrm{ET}(n=5,19 \%)$ and PV $(n=4,15 \%)(p<0.001)$. In a multivariate Cox regression model fitted on the whole cohort and adjusted for the wave to which patients belonged (Fig. 1B), significant independent risk factors for death were age over 70 years $(\mathrm{HR}=5.22,95 \% \mathrm{Cl} 1.80-15.14, p=0.002)$, male sex $(\mathrm{HR}=1.88,95 \% \mathrm{Cl} 1.13-3.13, p=0.016)$, severity of COVID19 defined by the need for respiratory support $(H R=4.45,95 \%$ $\mathrm{Cl} 1.85-10.70, p=0.001)$, and ruxolitinib discontinuation $(\mathrm{HR}=2.98$, $95 \% \mathrm{Cl} 1.29-6.89, p=0.011)$. Conversely, continuation of ruxolitinib was not a significant predictor ( $\mathrm{HR}=1.21, p=0.566)$.

Compared to the first wave, mortality in patients aged $60-70$ was reduced from 35 to $2 \%$. By contrast, deaths in patients over 70 years of age were recorded in $36 \%$ and $21 \%$ in the first and second wave, respectively. These patients, compared with those $<70$ years (Supplementary Table 1S), had more comorbidities, prior history of thrombosis, were more frequently hospitalized and in need of respiratory support. In these patients, deaths occurred in $59 \%, 23 \%$ and $18 \%$ of MF, ET and PV, respectively. Therefore, the benefit on survival was documented in patients younger than 70 years and more fit, with a limited degree of inflammation. 
Table 1. Patients' characteristics by the two waves of the COVID-19 pandemic.

\begin{tabular}{|c|c|c|c|}
\hline & $\begin{array}{l}\text { First wave } \\
N=175\end{array}$ & $\begin{array}{l}\text { Second wave } \\
N=304\end{array}$ & $p$ value \\
\hline \multicolumn{4}{|l|}{ MPN diagnosis } \\
\hline ET & $51(29.1 \%)$ & $110(36.2 \%)$ & 0.12 \\
\hline PV & $46(26.3 \%)$ & $89(29.3 \%)$ & 0.48 \\
\hline MF & $60(34.3 \%)$ & $74(24.3 \%)$ & 0.020 \\
\hline pre-PMF & $18(10.3 \%)$ & $31(10.2 \%)$ & 0.98 \\
\hline \multicolumn{4}{|c|}{ Treatments at last MPN f-up before Covid-19 } \\
\hline Cytoreduction & $141(80.6 \%)$ & $233(77.2 \%)$ & 0.38 \\
\hline Hydroxyurea & $79(56.0 \%)$ & $161(68.8 \%)$ & 0.092 \\
\hline Anagrelide & $8(5.7 \%)$ & $10(4.3 \%)$ & 0.48 \\
\hline Interferon & $4(2.8 \%)$ & 7 (3.0\%) & 0.99 \\
\hline Ruxolitinib & 45 (31.9\%) & $44(18.8 \%)$ & 0.002 \\
\hline Other & $5(3.5 \%)$ & $12(5.1 \%)$ & 0.53 \\
\hline ASA & 104 (59.4\%) & $180(59.4 \%)$ & 1.00 \\
\hline \multicolumn{4}{|c|}{ At Covid-19 diagnosis } \\
\hline Sex & & & 0.037 \\
\hline Female & $73(41.7 \%)$ & $158(52.0 \%)$ & \\
\hline Male & $102(58.3 \%)$ & $146(48.0 \%)$ & \\
\hline Age & $71.0(60.0-79.9)$ & $63.3(54.5-73.8)$ & $<0.001$ \\
\hline$<60$ yrs & $42(24.1 \%)$ & 117 (38.5\%) & \\
\hline $60-70$ yrs & 37 (21.3\%) & $83(27.3 \%)$ & \\
\hline$>70 \mathrm{yrs}$ & $95(54.6 \%)$ & $104(34.2 \%)$ & \\
\hline Patient disposition & & & $<0.001$ \\
\hline Home & $40(22.9 \%)$ & $208(68.4 \%)$ & \\
\hline Regular ward & $116(66.3 \%)$ & $88(28.9 \%)$ & \\
\hline ICU & 19 (10.9\%) & $8(2.6 \%)$ & \\
\hline $\begin{array}{l}\text { Respiratory } \\
\text { supplement need }\end{array}$ & $103(59.2 \%)$ & $83(27.6 \%)$ & $<0.001$ \\
\hline Not invasive & 99 (96.1\%) & $79(95.2 \%)$ & 0.75 \\
\hline Invasive & 20 (19.4\%) & 7 (8.5\%) & 0.037 \\
\hline $\mathrm{O}_{2}$ saturation $\%$ & $93.0(88.0-96.0)$ & $96.0(90.0-98.0)$ & $<0.001$ \\
\hline \multicolumn{4}{|l|}{ Symptoms } \\
\hline Fever & $141(80.6 \%)$ & $192(63.2 \%)$ & $<0.001$ \\
\hline Cough & $96(54.9 \%)$ & $132(43.4 \%)$ & 0.016 \\
\hline Dispnea & 98 (56.0\%) & $88(28.9 \%)$ & $<0.001$ \\
\hline Systemic & $36(20.6 \%)$ & $30(9.9 \%)$ & 0.001 \\
\hline Gastrointestinal & $22(12.6 \%)$ & $26(8.6 \%)$ & 0.16 \\
\hline Comorbidities & $130(74.3 \%)$ & $192(63.2 \%)$ & 0.012 \\
\hline Asthma & $3(1.7 \%)$ & $8(2.6 \%)$ & 0.53 \\
\hline Cerebrovascular & $23(13.2 \%)$ & $28(9.2 \%)$ & 0.18 \\
\hline Kidney impairment & 19 (10.9\%) & $5(1.7 \%)$ & $<0.001$ \\
\hline Heart disease & 25 (14.5\%) & 25 (8.3\%) & 0.035 \\
\hline COPD & $25(14.4 \%)$ & $19(6.3 \%)$ & 0.003 \\
\hline Smoke & 35 (23.0\%) & 47 (16.2\%) & 0.077 \\
\hline Hyperlipidemia & 47 (28.0\%) & $56(18.5 \%)$ & 0.018 \\
\hline Obesity & 21 (13.1\%) & $25(8.3 \%)$ & 0.098 \\
\hline Reumatic disease & $11(6.4 \%)$ & $13(4.3 \%)$ & 0.32 \\
\hline Hypertension & $104(60.8 \%)$ & $128(42.5 \%)$ & $<0.001$ \\
\hline Diabetes & $23(13.4 \%)$ & $27(8.9 \%)$ & 0.13 \\
\hline \multicolumn{4}{|l|}{ Chemistry } \\
\hline Hemoglobin $\mathrm{g} / \mathrm{dL}$ & $12.4(10.0-13.6)$ & $12.9(11.1-13.9)$ & 0.019 \\
\hline Hematocrit \% & $38.4(32.0-42.4)$ & $39.0(34.9-43.9)$ & 0.069 \\
\hline$W B C \times 10^{9} / L$ & $6.5(4.6-10.1)$ & $6.8(5.1-9.8)$ & 0.52 \\
\hline Neutrophils \% & $75.9(66.0-83.0)$ & $71.0(62.5-80.0)$ & 0.053 \\
\hline Lymphocytes \% & $14.0(9.0-20.0)$ & $17.6(10.2-25.0)$ & 0.022 \\
\hline $\mathrm{N} / \mathrm{L}$ ratio & $5.4(3.4-8.9)$ & $4.1(2.6-8.4)$ & 0.038 \\
\hline Platelets $\times 10^{9} / \mathrm{L}$ & $252.0(152.0-394.0)$ & $350.0(224.0-456.0)$ & $<0.001$ \\
\hline LDH U/L & $426.0(264.5-641.5)$ & $356.0(229.0-622.0)$ & 0.15 \\
\hline
\end{tabular}

Table 1. continued

\begin{tabular}{|c|c|c|c|}
\hline & $\begin{array}{l}\text { First wave } \\
N=175\end{array}$ & $\begin{array}{l}\text { Second wave } \\
N=304\end{array}$ & $p$ value \\
\hline CRP mg/L & $74.0(26.0-156.8)$ & $51.5(10.3-100.0)$ & 0.008 \\
\hline D-dimer $\mathrm{ng} / \mathrm{ml}$ & $801.0(398.0-1655.0)$ & $924.5(480.0-2340.0)$ & 0.20 \\
\hline \multicolumn{4}{|c|}{ Covid-directed treatments } \\
\hline Steroids & $45(27.8 \%)$ & $121(40.3 \%)$ & 0.007 \\
\hline Antibiotics & $114(70.4 \%)$ & $123(41.0 \%)$ & $<0.001$ \\
\hline Hydroxychloroquine & $100(60.2 \%)$ & $9(3.0 \%)$ & $<0.001$ \\
\hline Antivirals & $57(34.3 \%)$ & $19(6.4 \%)$ & $<0.001$ \\
\hline Experimentals & $19(11.2 \%)$ & $12(4.0 \%)$ & 0.002 \\
\hline Antithrombotics & $93(56.0 \%)$ & $114(38.4 \%)$ & $<0.001$ \\
\hline \multicolumn{4}{|c|}{ MPN-directed treatment change } \\
\hline $\begin{array}{l}\text { Hydroxyurea } \\
\text { discontinuation }\end{array}$ & $9(11.3 \%)$ & $11(6.6 \%)$ & 0.21 \\
\hline $\begin{array}{l}\text { Anagrelide } \\
\text { discontinuation }\end{array}$ & $1(12.5 \%)$ & $2(20.0 \%)$ & 0.67 \\
\hline $\begin{array}{l}\text { Interferon } \\
\text { discontinuation }\end{array}$ & $1(25.0 \%)$ & $1(14.3 \%)$ & 0.66 \\
\hline $\begin{array}{l}\text { Ruxolitinib } \\
\text { discontinuation }\end{array}$ & $11(23.9 \%)$ & $4(8.7 \%)$ & 0.048 \\
\hline \multicolumn{4}{|c|}{ Outcomes of the acute phase } \\
\hline Death & $50(28.6 \%)$ & $26(8.6 \%)$ & $<0.001$ \\
\hline Time to death (days) & $9.5(4-16)$ & $11.0(6-20)$ & 0.673 \\
\hline Thrombosis & $14(8.0 \%)$ & $5(1.6 \%)$ & 0.001 \\
\hline $\begin{array}{l}\text { Time to } \\
\text { thrombosis (days) }\end{array}$ & $11.5(4.0-25.0)$ & $1.0(1.0-6.0)$ & 0.52 \\
\hline Arterial & $3(1.7 \%)$ & $1(0.3 \%)$ & 0.141 \\
\hline Venous & $12(6.9 \%)$ & $4(1.3 \%)$ & 0.002 \\
\hline
\end{tabular}

Continuous variables are summarized by median (interquartile range [IQR]).

\section{THROMBOSIS}

At 60 days from COVID-19 diagnosis, only 5 incident cases of thrombosis were registered out of 304 patients $(1.6 \%)$ during the second wave, significantly lower than in the first wave (14 thrombosis on 175 patients, 8.0\%), although an antithrombotic treatment was prescribed less frequently (Table 1). Such findings mirror the less severe clinical presentation noticed in the present case series. However, almost all events $(n=4 / 5)$ were venous and we confirmed in multivariate model that most of these events occurred in patients with ET (SHR $=4.4,95 \% \mathrm{Cl} 1.8-10.7, p=$ 0.001 ). As in the first wave, we did not find a significant difference in venous thrombosis between cases treated with prophylactic doses of heparin compared to controls.

\section{EVENTS IN PATIENTS SURVIVING AFTER THE ACUTE PHASE}

Two-hundred twenty-three patients survived after the acute phase of the second wave of COVID-19 and were followed up for a median of 141 days (IQR: 94-173). Two of them died, 4 were diagnosed with deep vein thrombosis of the legs with or without pulmonary embolism and one with arterial cerebral thrombosis, and 4 developed bleeding, accounting together for an event-free survival (EFS) of $93.82 \%$, a figure significantly different from the first wave (EFS: $65.70 \%, p=0.0312$ ).

\section{COMMENT}

This is the largest analysis of MPN patients who contracted COVID19 in the 12 months subsequent to the first wave of the coronavirus pandemic, which was characterized by conditions of exceptional lethality. Patients of the second wave presented, compared to those of the first, with a less severe disease, including a lower degree of inflammation, leading to hospitalization in a 


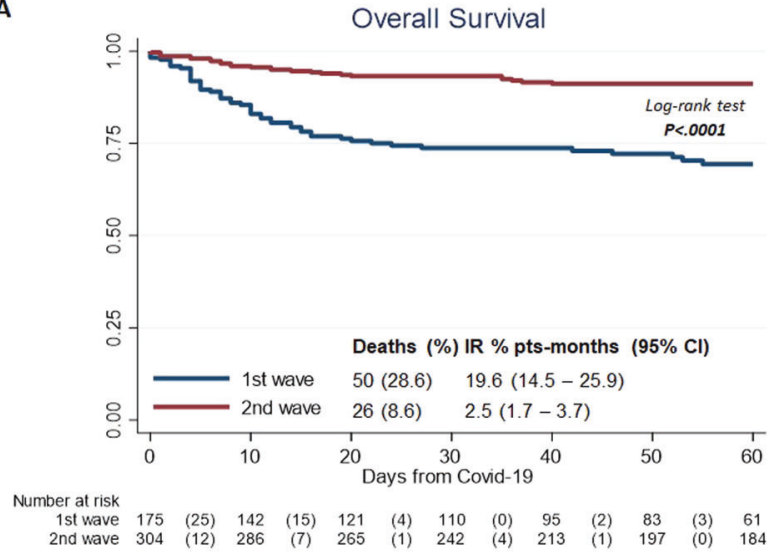

B

\begin{tabular}{|c|c|c|}
\hline & & \\
\hline $2^{\text {nd }}$ wave (vs. $1^{\text {st }}$ wave) & $.57(0.33-0.97)$ & 0.037 \\
\hline Male sex (vs. female) & $1.88(1.13-3.13)$ & 0.016 \\
\hline Comorbidities (at least one) & $1.19(0.61-2.31)$ & 0.615 \\
\hline \multicolumn{3}{|l|}{ Age at Covid-19 } \\
\hline$<60$ yrs & 1 (ref) & \\
\hline $60-70$ yrs & $2.44(0.78-7.65)$ & 0.126 \\
\hline$>70 \mathrm{yrs}$ & $5.22(1.80-15.14)$ & 0.002 \\
\hline \multicolumn{3}{|l|}{ MPN diagnosis } \\
\hline ET & 1 (ref) & \\
\hline PV & $.68(0.32-1.44)$ & 0.311 \\
\hline MF & $1.64(0.85-3.16)$ & 0.140 \\
\hline pre-PMF & $.49(0.16-1.49)$ & 0.207 \\
\hline \multicolumn{3}{|l|}{ Patient disposition } \\
\hline Home & 1 (ref) & \\
\hline Regular ward & $1.71(0.58-5.03)$ & 0.331 \\
\hline ICU & $3.30(0.96-11.33)$ & 0.058 \\
\hline Need of respiratory support & $4.45(1.85-10.70)$ & 0.001 \\
\hline Ruxolitinib administration & $1.21(0.63-2.34)$ & 0.566 \\
\hline Ruxolitinib discontinuation & $2.98(1.29-6.89)$ & 0.011 \\
\hline
\end{tabular}

Fig. 1 Overall survival by waves of coronavirus pandemic. Kaplan-Meier curves of overall survival by the two waves of Covid-19 (A) and multivariate Cox proportional hazard model for mortality during the acute phase (B).

smaller percentage of cases. Overall, the mortality rate was significantly lower, likely due to early COVID-19 diagnosis, facilitated by the greater availability of swabs than in the first wave, more efficient management of infected patients, better prepared health systems and preferential protection of older and higher-risk MPN vulnerable subjects. However, patients over 70 years still presented with an excess of mortality, particularly when associated with comorbidities and an MF phenotype. Unfortunately, no data are available so far in our series to support a role of vaccinations. The high thrombosis rate in patients with ET was confirmed, suggesting that in this MPN phenotype regimens of antithrombotic prophylaxis in addition to heparin should be explored. Also in the second wave, but to a lesser extent than in the first, the health consequences of COVID-19 protracted far beyond acute infection, suggesting careful and permanent surveillance of patients with MPN who have survived the acute phase of SARS-CoV-2 virus infection.

Tiziano Barbui $\mathbb{I D}^{1 凶}$, Alessandra lurlo $\mathbb{I D}^{2}$, Arianna Masciulli ${ }^{1}$, Alessandra Carobbio (iD ${ }^{1}$, Arianna Ghirardi ${ }^{1}$, Greta Carioli ${ }^{1}$, Marta Anna Sobas ${ }^{3}$, Elena Maria Elli ${ }^{4}$, Elisa Rumi (iD ${ }^{5}$ Valerio De Stefano ${ }^{6}{ }^{6}$, Francesca Lunghi ${ }^{7}$, Monia Marchetti ${ }^{8}{ }^{\prime}$, Rosa Daffini ${ }^{9}$, Mercedes Gasior Kabat ${ }^{10}$, Beatriz Cuevas (iD ${ }^{11^{\prime}}$, Maria Laura Fox ${ }^{12}$, Marcio Miguel Andrade-Campos ${ }^{13^{\prime}}$ Francesca Palandri (D) ${ }^{14}$, Paola Guglielmelli (iD) ${ }^{15}$, Giulia Benevolo ${ }^{16}$, Claire Harrison ${ }^{17}$, Maria Angeles Foncillas (iD ${ }^{18}$, Massimiliano Bonifacio (iD ${ }^{19}$, Alberto Alvarez-Larran ${ }^{20}$ Jean-Jacques Kiladjian ${ }^{21}$, Estefanía Bolaños Calderón ${ }^{22}$, Andrea Patriarca ${ }^{23}$, Keina Quiroz Cervantes (iD ${ }^{24}$, Martin Griessammer ${ }^{25}$, Valentin Garcia-Gutierrez (iD ${ }^{26}$ Alberto Marin Sanchez ${ }^{27}$, Elena Magro Mazo ${ }^{28}$, Marco Ruggeri ${ }^{29}$, Juan Carlos Hernandez-Boluda (iD ${ }^{30}$, Santiago Osorio ${ }^{31}$, Gonzalo Carreno-Tarragona (iD ${ }^{32}$, Miguel Sagues Serrano ${ }^{33^{\prime}}$ Rajko Kusec ${ }^{34}$, Begona Navas Elorza ${ }^{35}$, Anna Angona ${ }^{36}$, Blanca Xicoy Cirici ${ }^{37}$, Emma Lopez Abadia ${ }^{38}$, Steffen Koschmieder (iD ${ }^{39}$, Daniele Cattaneo (iD ${ }^{2,40}$, Cristina Bucelli ${ }^{\prime}$ Edyta Cichocka (1D ${ }^{41}$, Anna Masternak Kulikowska de Nałęcz ${ }^{42}$, Fabrizio Cavalca ${ }^{4}$, Oscar Borsani $\left(D^{5}\right.$, Silvia Betti ${ }^{6}$, Lina Benajiba ${ }^{21}$, Marta Bellini ${ }^{43}$, Natalia Curto-Garcia ${ }^{17}$ Alessandro Rambaldi $\mathbb{D}^{40,43}$ and Alessandro Maria Vannucchi ${ }^{15}$ ${ }^{1}$ FROM Research Foundation, Papa Giovanni XXIII Hospital, Bergamo, Italy. ${ }^{2}$ Hematology Division, Foundation IRCCS Ca' Granda Ospedale Maggiore Policlinico, Milan, Italy. ${ }^{3}$ Department of Hematology, Blood

Neoplasms and Bone Marrow Transplantation, Wroclaw Medical

University, Wrocław, Poland. ${ }^{4}$ Hematology Division and Bone Marrow Transplant Unit. San Gerardo Hospital, ASST Monza, Monza,
Italy. ${ }^{5}$ Department of molecular medicine, University of Pavia, Pavia, Italy. ${ }^{6}$ Section of Hematology, Department of Radiological and Hematological Sciences, Catholic University, Fondazione Policlinico

"A. Gemelli" IRCCS, Rome, Italy. 'IRCCS Ospedale San Raffaele, Milano, Italy. ${ }^{8}$ AOU SS.Antonio e Biagio e C.Arrigo, Alessandria, Italy. ${ }^{9}$ ASST-Spedali Civili, Brescia, Italy. ${ }^{10}$ Hospital Universitario La Paz, Madrid, Spain. ${ }^{11}$ Hospital Universitario de Burgos, Burgos, Spain.

${ }^{12}$ Department of Hematology, Vall d'Hebron Institute of Oncology (VHIO), Vall d'Hebron Hospital Universitari, Vall d'Hebron Barcelona Hospital Campus, C/ Natzaret, 115-117, 08035 Barcelona, Spain.

${ }^{13}$ Hospital del Mar, Barcelona, Spain. ${ }^{14}$ IRCCS Azienda OspedalieroUniversitaria di Bologna, Bologna, Italy. ${ }^{15}$ Center Research and Innovation of Myeloproliferative Neoplasms (CRIMM), Department of Experimental and Clinical Medicine, Azienda Ospedaliera Universitaria Careggi, University of Florence, Florence, Italy. ${ }^{16} \mathrm{AOU}$ Città della Salute e della Scienza di Torino, Torino, Italy. ${ }^{17}$ Guy's and

St. Thomas' NHS Foundation Trust, London, United Kingdom.

${ }^{18}$ Hospital Universitario Infanta Leonor, Madrid, Spain. ${ }^{19}$ Ospedale Policlinico "G.B. Rossi", Borgo Roma, Verona, Italy. ${ }^{20}$ Hospital Clinic de Barcelona, Barcelona, Spain. ${ }^{21}$ Hospital Saint-Louis, Paris, France.

${ }^{22}$ Hospital Clinico San Carlos, Madrid, Spain. ${ }^{23}$ AOU Maggiore della Carità, Novara, Italy. ${ }^{24}$ Hospital Universitario de Mostoles, Madrid, Spain. ${ }^{25}$ Oncology, Hemostaseology and Palliative Care, Johannes Wesling Medical Center, University Clinic for Hematology, Minden, Germany. ${ }^{26}$ Hospital Ramon y Cajal, IRYCIS, Madrid, Spain. ${ }^{27}$ Hospital General Universitario de Albacete, Albacete, Spain. ${ }^{28}$ Hospital Universitario Principe de Asturias, Alcalà de Henares, Madrid, Spain.

${ }^{29}$ Ospedale San Bortolo, Vicenza, Italy. ${ }^{30}$ Hospital Clinico Universitario, INCLIVA, Valencia, Spain. ${ }^{31}$ Hospital Gregorio Maranon, Madrid, Spain. ${ }^{32}$ Hospital Universitario 12 de Octubre, Madrid, Spain.

${ }^{33}$ ICO L'Hospitalet-Hospital Moises Broggi, Sant Joan Despì,

Barcelona, Spain. ${ }^{34}$ Department of haematology, Clinic of internal medicine, University Hospital Dubrava-School of Medicine University of Zagreb, Zagreb, Croatia. ${ }^{35}$ Hospital Moncloa, Madrid, Spain. ${ }^{36}$ ICO Girona Hospital Josep Trueta, Girona, Spain. ${ }^{37}$ Institut Català d' Oncologia-Hospital Germans Trias i Pujol, Josep Carreras Leukemia Research Institute, Universitat Autònoma de Barcelona, Barcelona, Spain. ${ }^{38}$ Hospital General de Elche, Elche (Alicante), Spain. ${ }^{39}$ Department of Hematology, Oncology, Hemostaseology, and Stem Cell Transplantation, Faculty of Medicine, RWTH Aachen University, Aachen, Germany. ${ }^{40}$ Università degli Studi di Milano, Milano, Italy.

${ }^{41}$ Department of Hematology and Bone Marrow Transplantation,

Nicolaus Copernicus Hospital, Torun, Poland. ${ }^{42}$ Department of Hematology and Internal Diseases, State Hospital, Opole, Poland.

${ }^{43}$ ASST Papa Giovanni XXIII, Bergamo, Italy. 凶email: tbarbui@fondazionefrom.it 


\section{REFERENCES}

1. Passamonti F, Cattaneo C, Arcaini L, Bruna R, Cavo M, Merli F, et al. Clinical characteristics and risk factors associated with COVID-19 severity in patients with haematological malignancies in Italy: a retrospective, multicentre, cohort study. Lancet Haematol. 2020;7:e737-45.

2. Jiménez $M$, Roldan E, Fernández-Naval C, Villacampa G, Martinez-Gallo M, MedinaGil D, et al. Cellular and humoral immunogenicity of the mRNA-1273 SARS-CoV-2 vaccine in patients with hematologic malignancies. Blood Adv. 2021. Online ahead of print as https://doi.org/10.1182/bloodadvances.2021006101.

3. Wood WA, Neuberg DS, Thompson JC, Tallman MS, Sekeres MA, Sehn LH, et al. Outcomes of patients with hematologic malignancies and COVID-19: a report from the ASH Research Collaborative Data Hub. Blood Adv. 2020;4:5966-75.

4. Barbui T, Vannucchi AM, Alvarez-Larran A, lurlo A, Masciulli A, Carobbio A, et al. High mortality rate in COVID-19 patients with myeloproliferative neoplasms after abrupt withdrawal of ruxolitinib. Leukemia. 2021;35:485-93.

5. Sheather SJ. Density estimation. Stat Sci. 2004;19:588-97.

\section{ACKNOWLEDGEMENTS}

The study was supported by a research grant by the COVID " $3 \times 1$ project", BREMBO S.p. A., Bergamo, Italy (TB) and by AIRC $5 \times 1000$ call "Metastatic disease: the key unmet need in oncology" to MYNERVA project, \#21267 (MYeloid NEoplasms Research Venture AIRC). A detailed description of the MYNERVA project is available at https://progettomynerva.it (AMV, PG). The study was also supported by HARMONY PLUS, which is funded through the Innovative Medicines Initiative (IMI), Europe's largest public-private initiative aiming to speed up the development of better and safer medicines for patients. The HARMONY Alliance has received funding from IMI 2 Joint Undertaking and is listed under grant agreement No. 945406. This Joint Undertaking receives support from the European Union's Horizon 2020 Research and Innovation Programme and the European Federation of Pharmaceutical Industries and Associations (EFPIA). IMI supports collaborative research projects and builds networks of industrial and academic experts in order to boost pharmaceutical innovation in Europe.

\section{AUTHOR CONTRIBUTIONS}

TB conceived and designed the study, supervised the analysis and wrote the paper. AMV, VDS, AR revised the study and contributed to manuscript writing. AM directed the project. $A C$ planned and performed statistical analyses. AG, GC contributed to dataset preparation. Al, MAS, EME, ER, FL, MM, RD, MGK, BC, MLF, MMAC, FP, PG, GB, $\mathrm{CH}, \mathrm{MAF}, \mathrm{MB}, \mathrm{AAL}, \mathrm{JJK}, \mathrm{EBC}, \mathrm{AP}, \mathrm{KSQC}, \mathrm{MG}, \mathrm{VGG}, \mathrm{AMS}, \mathrm{EMM}, \mathrm{MR}, \mathrm{JCHB}, \mathrm{SO}, \mathrm{GCT}$, MSS, RK, BNE, AA, BXC, ELA, SK, DC, CB, EC, AKdN, FC, OB, SB, LB, MB, NCG, collected data. All authors revised and approved the final version of the manuscript.

\section{COMPETING INTERESTS}

The authors declare no competing interests.

\section{ADDITIONAL INFORMATION}

Supplementary information The online version contains supplementary material available at https://doi.org/10.1038/s41375-022-01507-2.

Correspondence and requests for materials should be addressed to Tiziano Barbui.

Reprints and permission information is available at http://www.nature.com/ reprints

Publisher's note Springer Nature remains neutral with regard to jurisdictional claims in published maps and institutional affiliations. 